\title{
Validación y comparación de los puntajes CRUSADE y ACTION para predecir el riesgo de sangrado en pacientes con síndrome coronario agudo sin elevación del segmento ST
}

\author{
Validation and Comparison of the CRUSADE and \\ ACTION scores to predict the risk of bleeding in \\ patients with acute coronary syndrome without ST \\ segment elevation
}

\author{
Víctor Aldana, Daniel Vásquez, Juan Manuel Sénior, Arturo Rodríguez, \\ Andrés Fernández, Henry Borja, Gilma Hernández, Catalina Valencia, \\ Andrea Holguín • Medellín (Colombia)
}

\section{Resumen}

Objetivo: validar y comparar las escalas de riesgo de sangrado CRUSADE y ACTION en pacientes con síndrome coronario agudo sin elevación del segmento ST (SCASEST) llevados a estratificación coronaria invasiva temprana en un centro de alta complejidad.

Métodos: estudio de tipo observacional, analítico y prospectivo, de validación de escalas en una cohorte de pacientes con SCASEST llevados a estratificación coronaria invasiva temprana en un centro cardiovascular. Las escalas de riesgo CRUSADE y ACTION fueron calculadas con las características basales. La ocurrencia de sangrado mayor fue definido por hemorragia intracerebral, retroperitoneal, caída del hematocrito $\geq 12 \%$ o necesidad de transfusión cuando el hematocrito fuera $>3 \mathrm{~g} / \mathrm{dL}$ o $\geq 28 \%$ o $<28 \%$ con documentación de sangrado clínico o sangrado no relacionado con cirugía de revascularización.

Resultados: en este análisis la tasa de sangrado mayor observada fue de $4.8 \%$. La presencia de sangrado mayor se asoció con una probabilidad mayor de mortalidad durante el seguimiento a seis meses (OR 5.316; IC 95\% 1.77 -15.92; p= 0.002). La calibración de las escalas de riesgo fue adecuada de acuerdo al estadístico Hosmer-Lemeshow ( $\mathrm{p}>0.05$ ). Ambas escalas demostraron buena discriminación sin diferencias estadísticamente significativas en el escenario intrahospitalario (AUC ROC CRUSADE ACTION 0.77 vs 0.80 p=0.19), al mes (AUC ROC CRUSADE ACTION 0.70 vs $0.75 \mathrm{p}=0.08$ ) y 6 meses (AUC ROC CRUSADE ACTION 0.71 vs $0.76 \mathrm{p}=0.09$ ), respectivamente.

Conclusiones: las escalas de riesgo CRUSADE y ACTION son herramientas útiles para la predicción del riesgo de sangrado en pacientes con SCASEST llevados a estratificación coronaria invasiva temprana. Estos hallazgos sugieren el uso de la escala ACTION sobre la escala de riesgo CRUSADE. (Acta Med Colomb 2018; 43: 10-19).

Palabras clave: síndrome coronario agudo, sangrado, CRUSADE, ACTION

\begin{abstract}
Objective: to validate and compare the CRUSADE and ACTION bleeding risk scores in patients with acute coronary syndrome without ST-segment elevation (NSTE-ACS) undergoing early invasive coronary stratification in a highly complex center.

Methods: an observational, analytical and prospective study of scale validation in a cohort of patients with NSTEACS who underwent early invasive coronary stratification in a cardiovascular center. The CRUSADE and ACTION risk scales were calculated with the baseline characteristics. The occurrence of major bleeding was defined by intracerebral, retroperitoneal hemorrhage, he-
\end{abstract}

Dr. Víctor Aldana: Especialista en Medicina Interna y Cardiología. Fellow Cardiología Intervencionista, Hospital Universitario San Vicente Fundación, Universidad de Antioquia; Dr. Daniel Vásquez: Médico y Cirujano. Candidato a Magister en Ciencias Clínicas, Universidad de Antioquia; Dr. Juan Manuel Sénior Sánchez: Especialista en Medicina Interna y Cardiología. Especialista en Cardiología Intervencionista Hospital Universitario San Vicente de Paúl Fundación. Coordinador Posgrado Cardiología Clínica e Intervencionista Universidad de Antioquia. Coordinador Grupo para la Investigación de las Enfermedades Cardiovasculares; Dr. Arturo Rodríguez Dimuro: Especialista en Cardiología Intervencionista, Hospital Universitario San Vicente de Paúl Fundación, Universidad de Antioquia; Dr. Andrés Fernández Cadavid: Especialista en Cardiología Intervencionista, Hospital Universitario San Vicente de Paúl Fundación, Universidad de Antioquia; Dr. Henry Borja Romero: Especialista en Medicina Interna y Cardiología. Fellow Cardiología Intervencionista, Hospital Universitario San Vicente Fundación, Universidad de Antioquia; Dra. Gilma Hernández: Especialista en Estadística. Docente Facultad de Medicina, Universidad de Antioquia; Dra. Catalina Valencia Vera: Médico y Cirujano. Unidad de Dolor Torácico, Hospital Universitario San Vicente Fundación; Andrea Holguín Cardona: Estudiante Universidad de Antioquia. Medellín (Colombia).

Correspondencia: Dr. Juan Manuel Sénior. Medellín (Colombia).

E-mail: mmbt@une.net.co

Recibido: 18/VII/2016 Aceptado: 5/X/2017 
matocrit drop $\geq 12 \%$ or need for transfusion when the hematocrit was $>3 \mathrm{~g} / \mathrm{dL}$ or $\geq 28 \%$ or $<28 \%$ with clinical bleeding document or bleeding not related to revascularization surgery.

Results: in this analysis, the highest observed bleeding rate was $4.8 \%$. The presence of major bleeding was associated with a higher probability of mortality during six-month follow-up (OR $5.316,95 \%$ CI $1.77-15.92, p=0.002)$. The calibration of the risk scales was adequate according to the Hosmer-Lemeshow statistic ( $\mathrm{p}>0.05$ ). Both scales showed good discrimination without statistically significant differences in the intrahospital setting (AUC ROC CRUSADE ACTION 0.77 vs $0.80 \mathrm{p}=0.19$ ) one month (AUC ROC CRUSADE ACTION 0.70 vs $0.75 \mathrm{p}=0.08$ ) and 6 months (AUC ROC CRUSADE ACTION 0.71 vs $0.76 \mathrm{p}=0.09$ ), respectively.

Conclusions: the CRUSADE and ACTION risk scales are useful tools for the prediction of bleeding risk in patients with NSTEACS who are undergoing early invasive coronary stratification. These findings suggest the use of the ACTION scale over the CRUSADE risk scale. (Acta Med Colomb 2018; 43: 10-19)

Keywords: acute coronary syndrome, bleeding, CRUSADE, ACTION

\section{Introducción}

La presencia de sangrado en pacientes con síndrome coronario agudo sin elevación del segmento ST está asociado con un alto riesgo de mortalidad y nuevos eventos cardiovasculares mayores adversos. Debido a estas implicaciones clínicas, la estratificación del riesgo de sangrado se ha convertido en la actualidad en un componente primordial en el manejo de pacientes con síndrome coronario agudo $(1,2)$. El advenimiento de nuevas terapias antitrombóticas, en algunos casos ha venido de la mano con el aumento en las tasas de sangrado, impactando de forma negativa los desenlaces cardiovasculares $(3,4)$. De la misma manera ciertos esquemas de manejo han demostrado disminución significativa de los eventos isquémicos sin repercutir adversamente en las tasas de sangrado mayor (5-7). Dada la importancia de mantener los perfiles terapéuticos de seguridad y eficacia, el American College of Cardiology (ACC), la American Heart Association (AHA), European Cardiology Society (ECS), han perfeccionado nuevas pautas internacionales en la renovación del énfasis de estratificación de riesgo para guiar el tratamiento de pacientes con síndrome coronario agudo sin elevación del segmento ST $(8,9)$. CRUSADE bleeding score y ACTION Registry-GWTG risk score, han sido desarrolladas y validadas recientemente para evaluar el riesgo de sangrado en este escenario de pacientes $(10,11)$. Son limitadas las publicaciones en Colombia en la validación y comparación de estas escalas de riesgo $(12,13)$ y en Hispanoamérica (14); incluso, son escasas las validaciones en pacientes con síndrome coronario agudo sin elevación del segmento ST llevados a estratificación temprana predominantemente transradial (15).

En el presente estudio se buscó validar y comparar las escalas CRUSADE y ACTION en pacientes con síndrome coronario agudo sin elevación del segmento ST (SCASEST) para predecir el riesgo de sangrado, llevados a estratificación coronaria invasiva temprana en un centro de alta complejidad cardiovascular.

\section{Tipo de estudio}

\section{Métodos}

Se realizó un estudio de tipo observacional, analítico y prospectivo, de validación de escalas de riesgo de sangrado en una cohorte de pacientes mayores de 18 años con SCASEST (angina inestable o infarto agudo de miocardio sin elevación del ST) atendidos en la unidad de cuidados coronarios de una institución de alta complejidad de la ciudad de Medellín.

\section{Población objetivo}

Pacientes con diagnóstico de síndrome coronario agudo sin elevación del segmento ST, mayores de 18 años, ingresados en forma consecutiva a la unidad de cuidados coronarios de una institución de alta complejidad, en el periodo comprendido entre enero de 2013 y noviembre de 2014.

\section{Criterios de elegibilidad}

Criterios de inclusión. Todos los pacientes que ingresaron a la institución con diagnóstico de síndrome coronario agudo sin elevación del segmento ST mayores de 18 años de edad, que se presentaron con angina típica clasificada como inestable por las siguientes características: angina en reposo de más de 20 minutos de duración en las 48 horas previas al ingreso, de reciente comienzo (últimos 60 días) $\mathrm{o}$ in crescendo (que haya cambiado un estado funcional de la clasificación canadiense de angina y sea mínimo estado III), más uno de los siguientes: marcadores enzimáticos de necrosis positivos definidos como elevación de troponina sérica por encima del percentil 99 y/o cambios electrocardiográficos de isquemia, definidos como inversión de la onda $\mathrm{T}$ profunda $\geq 0.1 \mathrm{mV}$, infradesnivel del ST mayor a $1 \mathrm{~mm}$ o elevación transitoria (menor a 20 minutos) del ST de más 80 mseg de duración en más de dos derivaciones continuas (16).

Criterios de exclusión. Pacientes quienes previo al ingreso a la institución se encontraran en manejo con medicación anticoagulante oral o aquellos con muerte de cualquier origen dentro de las primeras 48 horas del ingreso o expectativa 
de vida menor a los seis meses. También, pacientes que no cumplieran con el seguimiento clínico para el análisis de desenlaces adversos o se rehusaran a participar.

\section{Selección y tamaño de la muestra}

La aproximación convencional al cálculo de tamaño de muestra para modelos predictivos define un valor entre $5 \mathrm{y}$ 20 desenlaces por cada variable independiente. Sin embargo, para la indicación específica de comparación de modelos predictivos no existe evidencia que sustente la anterior consideración, aunque algunos autores sugieren un número similar de desenlaces y no desenlaces. Dado que el objetivo general del presente estudio partió de la hipótesis de una mayor exactitud de una escala versus la otra, se tomaron como referencia los valores originales de áreas bajo la curva ROC (AUC-ROC) de los dos modelos CRUSADE (0.70) Y ACTION (0.74). Para probar diferencias entre dos áreas bajo la curva (AUC-ROC), se utiliza la fórmula de Hanley-Mc Neil y Obuchowsky-Mclish, para la cual se eligieron en este estudio una probabilidad de error tipo I de 0.05 , potencia de la prueba de 0.8 y correlación entre las dos escalas de 0.84 . La ecuación utilizada fue:

$$
\mathrm{n}=\frac{\left[\mathrm{Z} \frac{\alpha}{2} \sqrt{V_{0}\left(\theta_{1}-\theta_{2}\right)}+Z_{\beta} \sqrt{V_{0}\left(\theta_{1}-\theta_{2}\right)}\right]^{2}}{\left(\theta_{1}-\theta_{1}\right)}
$$

donde

$$
V\left(\theta_{1}-\theta_{1}\right)=n \operatorname{Var}\left(\theta_{1}+n \operatorname{Var}\left(\theta_{2}\right)-2 n \operatorname{Cov}\left(\theta_{1}, \theta_{2}\right)\right.
$$

Y

$$
\begin{gathered}
V\left(\theta_{0}\right)=f^{2}\left[1+\frac{B^{2}}{R}+\frac{A^{2}}{2}\right]+g^{2}\left[\frac{B^{2}(1+R)}{2 R}\right] \\
C\left(\theta_{1}, \theta_{2}\right)=f_{1} f_{2}\left[r_{D}+\frac{r_{N D} B_{1} B_{2}}{R}+\frac{r_{D}{ }_{D} A_{1} A_{2}}{2}\right]+g_{1} g_{2}\left[\frac{B_{1} B_{2}\left(r^{2}{ }_{N D}+R r^{2}{ }_{D}\right)}{2 R}\right] \\
+f_{1} g_{2} \frac{\left[r^{2}{ }_{D} A_{1} B_{2}\right]}{2}+f_{2} g_{1} \frac{\left[r^{2}{ }_{D} A_{2} B_{1}\right]}{2}
\end{gathered}
$$

Con esto se encontró un tamaño de muestra de 246 individuos por cada una de las escalas, para un total de 492 sujetos.

\section{Plan de Reclutamiento}

Se reclutaron todos los pacientes que cumplieron con los criterios de inclusión en el periodo de tiempo determinando y hasta completar el tamaño de muestra calculado; a todos se les calculó el puntaje basal de riesgo de sangrado CRUSADE y ACTION al momento del ingreso a la institución.

Los pacientes se evaluaron al mes y a los seis meses luego de la hospitalización por el evento coronario agudo índice, con controles clínicos y telefónicos en los cuales se evaluaron los eventos cardiovasculares mayores adversos de interés.

\section{Procedimiento para la recolección de la información}

Se elaboró una base de datos en el programa Acces ${ }^{\circledR}$ para la recolección de los datos en forma prospectiva y de acuerdo a los modelos de predicción de las dos escalas.

Se estratificaron los pacientes en cinco categorías de riesgo de sangrado mayor durante la hospitalización según los puntos de corte establecidos por el estudio CRUSADE y el ACTION: muy bajo, < 20 puntos; bajo, 21 - 30 puntos; moderado, 31-40 puntos; alto 41-50 puntos; $y$ muy alto, $>50$ puntos. Posteriormente uno de los investigadores principales tabuló la información lograda en la base de datos para registro y archivo y el análisis estadístico se realizó con el paquete STATA 13.

\section{Plan de análisis de los resultados}

Análisis Estadístico. Se describieron las características clínicas, paraclínicas y sociodemográficas de los pacientes con SCASEST, utilizando métodos estadísticos acorde con la naturaleza de las variables. Para las variables categóricas se obtuvieron distribuciones de frecuencias y porcentajes y para las cuantitativas se calcularon las medidas de tendencia central y dispersión de acuerdo con la forma de la distribución, para lo cual se utilizó prueba de bondad de ajuste para normalidad de Shapiro Wilks. Finalmente se obtuvieron los estadísticos descriptivos de la estratificación según los valores resultados de las escalas.

Para determinar la asociación entre mortalidad general y sangrado intrahospitalario, al mes y a los seis meses posintervención, se utilizaron las pruebas Chi Cuadrado de independencia o test exacto de Fisher según la información obtenida. Se consideró un nivel de significación de 0.05 para estas pruebas.

Para la validación de los modelos predictivos, se utilizaron las mismas estrategias que en el estudio original. Inicialmente se validaron los modelos de predicción clínica utilizados en los puntajes CRUSADE y ACTION en población de Medellín provenientes de una institución de alta complejidad. Para ello se utilizó el modelo de regresión binaria logística y las variables predictoras para CRUSADE intrahospitalario fueron: hematocrito, sexo, frecuencia cardiaca, presión arterial sistólica, signos y síntomas de falla cardiaca, presencia de diabetes mellitus, enfermedad arterial periférica como comorbilidad, y cálculo de la depuración de creatinina, mientras que para ACTION fueron: edad, sexo, peso, frecuencia cardiaca, presión arterial sistólica, hemoglobina, creatinina, signos y síntomas de falla cardiaca, cambios del segmento ST, diabetes mellitus y enfermedad arterial periférica como comorbilidad, sin considerarse el consumo de warfarina domiciliaria; así mismo para la validación al mes y a los seis meses se incluyeron estas mismas variables.

Para cada modelo se obtuvieron estadísticos para validar la calibración y la capacidad discriminativa. En el primer caso, se usó el estadístico de Hosmer y Lemeshow para determinar si el modelo logístico ajustaba bien a los datos y para la discriminación se calcularon las áreas bajo la curva 
AUC-ROC en cada modelo con el respectivo gráfico, considerando en este caso un valor de esta área mayor o igual a 0.7 para relevancia clínica.

Finalmente, para la comparación de la capacidad predictiva de las dos escalas, se llevó a cabo una prueba estadística que compara áreas bajo la curva AUC-ROC, con el algoritmo de DeLong, DeLong y Pearson. La comparación se realizó de forma intrahospitalaria, al mes y seis meses, con el desenlace de sangrado. Para estas pruebas se usó nivel de significación estadística de 0.05 .

\section{Resultados}

Se determinó en 552 pacientes con diagnóstico de ingreso de síndrome coronario agudo sin elevación del segmento ST el riesgo de sangrado intrahospitalario, a los 30 días y a los seis meses del alta médica. Se incluyeron 507 individuos; 45 pacientes fueron excluidos, 43 por presentar elevación persistente del segmento ST y dos pacientes cuyo diagnóstico final fue disección aórtica (Figura 1). El promedio de edad fue de $65 \pm 11$ años, siendo el $54.4 \%$ mayor de 65 años predominando en $55.6 \%$ el sexo masculino, con un peso comprendido de $68.3 \pm 12.8 \mathrm{Kg}$ donde $43.8 \%$ tenía al menos tres factores de riesgo coronario. El tipo de síndrome coronario predominante al ingreso hospitalario fue angina inestable en $52.3 \%$, por su parte el infarto de miocardio sin elevación del segmento ST se presentó en $47.7 \%$ de los casos, con distribuciones similares a las del registro CRUSADE y estudio ACTION (Tabla 1).

\section{Manejo intrahospitalario}

Se registraron las cifras de frecuencia cardiaca y presión arterial de los pacientes al ingreso, siendo $79 \pm 20 \mathrm{lpm}$ y $138 \pm 27 \mathrm{mmHg}$ respectivamente, al igual que la presencia de diabetes mellitus (30\%), enfermedad arterial periférica (7.5\%) y de signos y síntomas de falla cardiaca (12.1\%). Se obtuvieron los siguientes resultados de los paraclínicos solicitados: hemoglobina inicial de $13.8 \pm 2 \mathrm{~g} / \mathrm{dL}$, hematocrito de $40.9 \pm 5.6 \%$, creatinina de $1.6 \pm 5.14 \mathrm{mg} / \mathrm{dL}$, y depuración promedio de $67.8 \pm 30.5 \mathrm{~mL} / \mathrm{min}$. El $6.5 \%$ tuvo complicaciones relacionadas al síndrome coronario agudo, $4.5 \%$ de tipo eléctricas y $2 \%$ mecánicas. Del total de pacientes tan sólo $2.2 \%$ no se le realizó angiografía coronaria y se les dio manejo médico. De los 496 pacientes estratificados en forma invasiva, $78 \%$ ameritó intervención coronaria percutánea, $69.8 \%$ tenía lesión en al menos una arteria coronaria mayor de $50 \%, 12.4 \%$ requirió cirugía de puentes, mientras el $9.6 \%$ recibió solo manejo médico. El 6.3\% tuvo alguna complicación relacionada con la angiografía, de las cuales $2.8 \%$ fue hematoma del sitio de punción, $1.6 \%$ desarrollo nefrotoxicidad, seudoaneurisma $0.4 \%$, ruptura arterial (radial) $0.4 \%$ y disección $0.2 \%$.

Los medicamentos prescritos durante la hospitalización y al alta fueron los siguientes: $97.9 \%$ recibió un inhibidor del receptor P2Y12, 94.5\% clopidogrel, $0.2 \%$ prasugrel y $3.2 \%$ ticagrelor; 97.4\% ASA; el 16\% inhibidor de glicoproteína

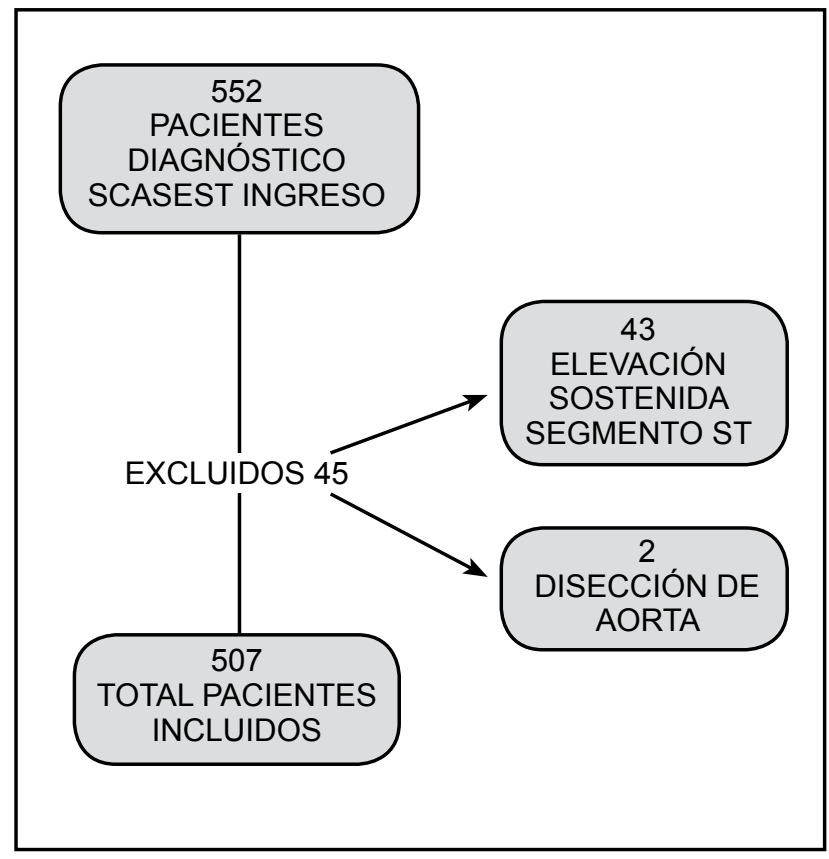

Figura 1. Pacientes con síndrome coronario agudo sin elevación del segmento ST.

IIb/IIIa (tirofiban). Fue indicado para el $92.1 \%$ un IECA/ ARA II; el $92.9 \%$ un betabloqueador (metoprolol o carvedilol), nitratos en el 33.5\%, 96.4\% estatinas, $47.1 \%$ diuréticos, $30.2 \%$ un calcioantagonista y $24.5 \%$ un antialdosterónico. En cuanto a la anticoagulación se utilizó: enoxaparina 93.3\%, heparina intravenosa $30.6 \%$ y fondaparinux tan sólo en $4.3 \%$. Otros medicamentos menos manejados fueron: digoxina $2.2 \%$, inotrópicos $25.4 \%$ y vasopresores $7.5 \%$.

\section{Estratificación de riesgo por las escalas CRUSADE y ACTION}

Se clasificó a los 507 pacientes de la cohorte según la escala CRUSADE en riesgo muy bajo $43 \%$, bajo $21.7 \%$, moderado $16.8 \%$, alto $9.8 \%$ y muy alto riesgo $8.7 \%$. De acuerdo con los parámetros de este puntaje, se presentó sangrado significativo, según las definiciones establecidas, en $2.3 \%$ de los pacientes estratificados en muy bajo riesgo, en $4.6 \%$ de bajo riesgo, el $3.6 \%$ de riesgo moderado, $12 \%$ de alto riesgo y $11.4 \%$ de muy alto riesgo. De acuerdo con la escala ACTION la cohorte de pacientes se estratificó en riesgo muy bajo $23.9 \%$, bajo $52 \%$, moderado $19.3 \%$, alto $4.2 \%$ y muy alto riesgo $0.6 \%$. Con base en esta distribución, el porcentaje de sangrado en cada uno fue de $1.7 \%$ en muy bajo riesgo, $4.2 \%$ en riesgo bajo, $8.2 \%$ en riesgo moderado, $14.2 \%$ de los pacientes con riesgo alto presentaron sangrado y ninguno de los pacientes clasificados en muy alto riesgo presentaron sangrado.

\section{Desenlaces}

Intrahospitalario. La distribución de sangrado por cualquier causa es similar en los diversos grupos de riesgo con diferencias significativas tanto para el CRUSADE ( $\mathrm{chi}^{2}$ 
Tabla 1. Características de la población al ingreso utilizadas en el modelo.

\begin{tabular}{|c|c|c|c|}
\hline Característica & $\begin{array}{c}\text { Cohorte validación } \\
\text { (507) }\end{array}$ & $\begin{array}{c}\text { CRUSADE } \\
(\mathbf{1 7 8 5 7})^{*}\end{array}$ & $\begin{array}{l}\operatorname{ACTION} \\
(\mathbf{1 0 8 2 1})^{* *}\end{array}$ \\
\hline \multicolumn{4}{|l|}{ Demográficas } \\
\hline Edad (años) & $65(28-93)$ & $67.0(56.0-79.0)$ & $64.0(54.0-76.0)$ \\
\hline Sexo masculino (\%) & 55.6 & 60.3 & 64.7 \\
\hline Peso $(\mathrm{Kg})$ & $68.6(35-115)$ & $81.1(68.1-95.3)$ & $83.0(71.0-97.0)$ \\
\hline \multicolumn{4}{|l|}{ Historia médica } \\
\hline Angina (\%) & 65.3 & 27.9 & 7.6 \\
\hline Cirugía de puentes (\%) & 6.1 & 18.5 & 14.3 \\
\hline Falla cardiaca $(\%)$ & 12.4 & 16.1 & 15.4 \\
\hline Diabetes $(\%)$ & 30 & 32.5 & 28.8 \\
\hline Dislipidemia (\%) & 53.5 & 51.7 & 55.3 \\
\hline Hipertensión (\%) & 76.9 & 70.6 & 68.5 \\
\hline Enfermedad coronaria $(\%)$ & 33.9 & 33.9 & \\
\hline Intervención coronaria percutánea (\%) & 17.9 & 20.5 & 22.7 \\
\hline Enfermedad arterial periférica (\%) & 7.5 & 18.1 & 9.3 \\
\hline Tabaquismo (\%) & 51.3 & 27.8 & - \\
\hline \multicolumn{4}{|l|}{ Ingreso } \\
\hline Pulso & $79(37-200)$ & $83.0(70-98)$ & $80(68.0-96.0)$ \\
\hline Presión arterial sistólica & $138(66-250)$ & $144(124-165)$ & $142(121,161)$ \\
\hline Hematocrito & $43.5(39.5-47.6)$ & $40.7(36.6-44.1)$ & - \\
\hline Signos de falla cardiaca congestiva (\%) & 12.1 & 23.2 & 15.4 \\
\hline Creatinina basal (mg/dL) & $1.6(0.4-90)$ & $1.02(0.90-1.25)$ & $1.1(0.9,1.3)$ \\
\hline Depuración de creatinina*** & $67,9(65.3-70.6)$ & $70.8(44.0-102.0)$ & - \\
\hline Cambios electrocardiográficos (\%) & 41.8 & 27.6 & $17.4 \%$ \\
\hline Uso previo de ASA (\%) & 46.4 & - & 43.4 \\
\hline Tipo de síndrome coronario agudo angina inestable/infarto sin ST (\%) & $52.3 / 47.7$ & & \\
\hline \multicolumn{4}{|c|}{$\begin{array}{l}\text { *Tomado de Baseline risk of major bleeding in non-ST-segment-elevation myocardial infarction: the CRUSADE (Can Rapid risk stratification of Unstable angina patients Suppress ADv } \\
\text { outcomes with Early implementation of the ACC/AHA Guidelines) Bleeding Score. } \\
\text { ** In-hospital major bleeding during st-elevation and nonst-elevation myocardial infarction care: Derivation and validation of a model from the action registry@-GWTGTM. } \\
\text { ***alculado con la fórmula Cockcroft-Gault }{ }^{17}\end{array}$} \\
\hline
\end{tabular}

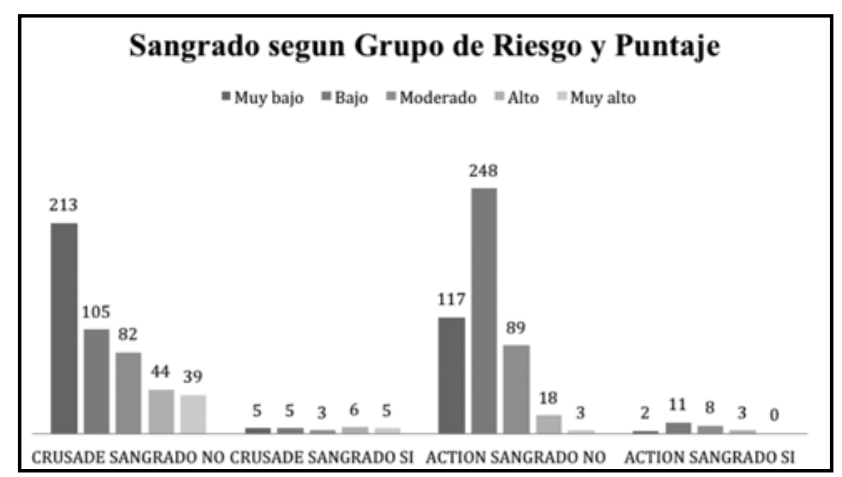

Figura 2. Presencia de sangrado según puntaje en función del tipo de riesgo al ingreso hospitalario. homogeneidad 13.371 y p=0.0096, $4 \mathrm{gl} \mathrm{chi}^{2}$ de tendencia 10.41 y p=0.013, $1 \mathrm{gl})$, como para el ACTION (chi' homogeneidad 6.672 y p=0.15, $4 \mathrm{gl}$; chi $^{2}$ de tendencia 5.342 y $\mathrm{p}=0.02,1 \mathrm{gl}$ ) (Figura 1). Se identificaron $24(4.8 \%)$ pacientes que sangraron (Figura 2).

Al mes. Del desenlace de interés, se presentó sangrado en seis pacientes representando un $1.3 \%$. La distribución de sangrado es similar en los diversos grupos de riesgo, sin diferencias significativas, para el CRUSADE $\left(\mathrm{chi}^{2}\right.$ homogeneidad 3.483 y $\mathrm{p}=0.48,4 \mathrm{gl} ; \mathrm{chi}^{2}$ de tendencia $0.75 \mathrm{y}$ $\mathrm{p}=0.38,1 \mathrm{gl})$, pero si para el ACTION $\left(\mathrm{chi}^{2}\right.$ homogeneidad 6.672 y $\mathrm{p}=0.15,4 \mathrm{gl} ; \mathrm{chi}^{2}$ de tendencia $5,342 \mathrm{y} \mathrm{p}=0.02,1$ gl) (Tablas 2 y 3 ). 
TRABAJOS ORIGINALES • Predicción del riesgo de sangrado en pacientes con síndrome coronario agudo

Tabla 2. Estratificación de riesgo de sangrado según el puntaje CRUSADE a los 30 días del alta médica.

\begin{tabular}{|c|c|c|c|c|c|c|}
\hline \multirow[b]{2}{*}{ Sangrado 30 días } & \multicolumn{5}{|c|}{ CRUSADE puntaje riesgo } & \\
\hline & 1 & 2 & 3 & 4 & 5 & \\
\hline No & 204 & 103 & 77 & 50 & 40 & $474(98.7 \%)$ \\
\hline \multirow[t]{2}{*}{$\mathrm{Si}$} & 1 & 2 & 2 & 0 & 1 & $6(1.3 \%)$ \\
\hline & $\begin{array}{c}205 \\
(42.7 \%)\end{array}$ & $\begin{array}{c}105 \\
(21.9 \%)\end{array}$ & $\begin{array}{c}79 \\
(16.5 \%)\end{array}$ & $\begin{array}{c}50 \\
(10.4 \%)\end{array}$ & $\begin{array}{c}41 \\
(8.5 \%)\end{array}$ & 480 \\
\hline
\end{tabular}

\begin{tabular}{|l|c|}
\hline Chi-cuadrado & 3.483 \\
\hline Grados de libertad & 4 \\
\hline Nivel de significancia & $\mathrm{P}=0.4804$ \\
\hline Chi cuadrado de tendencia & 0.755 \\
\hline Chi cuadrado (tendencia) & 1 \\
\hline Grados de libertad & $\mathrm{P}=0.3848$ \\
\hline Nivel de significancia &
\end{tabular}

Tabla 3. Estratificación de riesgo de sangrado según el puntaje ACTION a los 30 días del alta médica.

\begin{tabular}{|c|c|c|c|c|c|c|}
\hline \multirow[b]{2}{*}{ Sangrado 30 días } & \multicolumn{5}{|c|}{ ACTION puntaje riesgo } & \\
\hline & 1 & 2 & 3 & 4 & 5 & \\
\hline No & 114 & 245 & 87 & 20 & 1 & $467(98.7 \%)$ \\
\hline \multirow[t]{2}{*}{$\mathrm{Si}$} & 1 & 3 & 2 & 0 & 0 & $6(1.3 \%)$ \\
\hline & $\begin{array}{c}115 \\
(24.3 \%)\end{array}$ & $\begin{array}{c}248 \\
(52.4 \%)\end{array}$ & $\begin{array}{c}89 \\
(18.8 \%)\end{array}$ & $\begin{array}{c}20 \\
(4.2 \%)\end{array}$ & $\begin{array}{c}1 \\
(0.2 \%)\end{array}$ & 473 \\
\hline
\end{tabular}

\begin{tabular}{|l|c|}
\hline Chi-cuadrado & 1.103 \\
\hline Grados de libertad & 4 \\
\hline Nivel de significancia & $\mathrm{P}=0.8937$ \\
\hline Chi cuadrado de tendencia & 0.168 \\
\hline Chi cuadrado (tendencia) & 1 \\
\hline Grados de libertad & $\mathrm{P}=0.6819$ \\
\hline Nivel de significancia &
\end{tabular}

A los seis meses. El desenlace de interés se presentó en siete pacientes representando una frecuencia de $1.6 \%$. La distribución de sangrado es similar en los diversos grupos de riesgo, sin diferencias significativas tanto para el CRUSADE (chi ${ }^{2}$ homogeneidad 4.177 y p $=0.38,4 \mathrm{gl}$; $\mathrm{chi}^{2}$ de tendencia 1.143 y $\mathrm{p}=0.28,1 \mathrm{gl})$, como para el ACTION $\left(\mathrm{chi}^{2}\right.$ homogeneidad $1.015 \mathrm{y} \mathrm{p}=0.90,4 \mathrm{gl} ; \mathrm{chi}^{2}$ de tendencia 0.13 y $\mathrm{p}=0.71,1 \mathrm{gl})$ (Tablas 4 y 5$)$.

\section{Relación entre sangrado y mortalidad}

De 507 pacientes 24 presentaron sangrado en el escenario intrahospitalario, de los cuales falleció uno (OR 1 IC 95\% 0.12-7.8); a los 30 días el OR fue de 4.53 (IC 95\% 1.0319.91; $\mathrm{p}=0.045$ ) y a los seis meses el OR fue de 5.316 (IC 95\% 1.77-15.92; $\mathrm{p}=0.0028)$.

\section{Calibración y discriminación}

Desenlace sangrado. La calibración del modelo fue adecuada en la población de estudio de acuerdo al estadís- tico de Hosmer Lemeshow (Tabla 6). La discriminación del desenlace de sangrado fue buena para ambas escalas en el escenario intrahospitalario, al mes y a seis meses, demostrándose diferencias no significativas en la capacidad predictiva de los modelos; sin embargo, el modelo de predicción clínica ACTION en los distintos escenarios presenta un AUC-ROC mayor a 0.75 (Figuras 3-5).

\section{Discusión}

En el presente estudio, se ha evaluado la capacidad predictiva de dos escalas de riesgo de sangrado en pacientes con SCASEST, encontrando algunas diferencias en las características basales en la población estudiada con respecto a las reportadas en las escalas CRUSADE y ACTION. En la muestra el peso fue en promedio de $68.6 \mathrm{~kg}$ vs la población de CRUSADE y ACTION que fue de $81.1 \mathrm{~kg}$ y 83.0 $\mathrm{kg}$, respectivamente; el $51.3 \%$ de nuestra población tenía antecedente de tabaquismo, porcentaje mayor al reportado en el CRUSADE, en el cual fue de tan sólo $27.8 \%$, igual- 
Tabla 4. Estratificación de riesgo de sangrado según el puntaje CRUSADE a los seis meses del alta médica.

\begin{tabular}{|c|c|c|c|c|c|c|}
\hline \multicolumn{7}{|c|}{ CRUSADE puntaje riesgo } \\
\hline $\begin{array}{c}\text { Sangrado seis } \\
\text { meses }\end{array}$ & 1 & 2 & 3 & 4 & 5 & \\
\hline No & 196 & 98 & 74 & 46 & 37 & $451(98,5 \%)$ \\
\hline \multirow[t]{2}{*}{$\mathrm{Si}$} & 2 & 2 & 0 & 2 & 1 & $7(1,5 \%)$ \\
\hline & $\begin{array}{c}198 \\
(43.2 \%)\end{array}$ & $\begin{array}{c}100 \\
(21.8 \%)\end{array}$ & $\begin{array}{c}74 \\
(16.2 \%)\end{array}$ & $\begin{array}{c}48 \\
(10.5 \%)\end{array}$ & $\begin{array}{c}38 \\
(8.3 \%)\end{array}$ & 458 \\
\hline
\end{tabular}

\begin{tabular}{|l|c|}
\hline Chi-cuadrado & 4.177 \\
\hline Grados de libertad & 4 \\
\hline Nivel de significancia & $\mathrm{P}=0.3826$ \\
\hline Chi cuadrado de tendencia & 1.143 \\
\hline Chi cuadrado (tendencia) & 1 \\
\hline Grados de libertad & $\mathrm{P}=0.2851$ \\
\hline Nivel de significancia & \\
\hline
\end{tabular}

Tabla 5. Estratificación de riesgo de sangrado según el puntaje ACTION a los seis meses del alta médica.

\begin{tabular}{|c|c|c|c|c|c|c|}
\hline \multirow[b]{2}{*}{ Sangrado 30 días } & \multicolumn{5}{|c|}{ ACTION puntaje riesgo } & \\
\hline & 1 & 2 & 3 & 4 & 5 & \\
\hline No & 110 & 231 & 83 & 19 & 1 & $444(98.4 \%)$ \\
\hline $\mathrm{Si}$ & 1 & 4 & 2 & 0 & 0 & $7(1.6 \%)$ \\
\hline & $\begin{array}{c}111 \\
(24.6 \%)\end{array}$ & $\begin{array}{c}235 \\
(52.1 \%)\end{array}$ & $\begin{array}{c}85 \\
(18.8 \%)\end{array}$ & $\begin{array}{c}19 \\
(4.2 \%)\end{array}$ & $\begin{array}{c}1 \\
(0.2 \%)\end{array}$ & 451 \\
\hline
\end{tabular}

\begin{tabular}{|l|c|}
\hline Chi-cuadrado & 1.015 \\
\hline Grados de libertad & 4 \\
\hline Nivel de significancia & $\mathrm{P}=0.9076$ \\
\hline Chi cuadrado de tendencia & 0.137 \\
\hline Chi cuadrado (tendencia) & 1 \\
\hline Grados de libertad & $\mathrm{P}=0.7110$ \\
\hline Nivel de significancia &
\end{tabular}

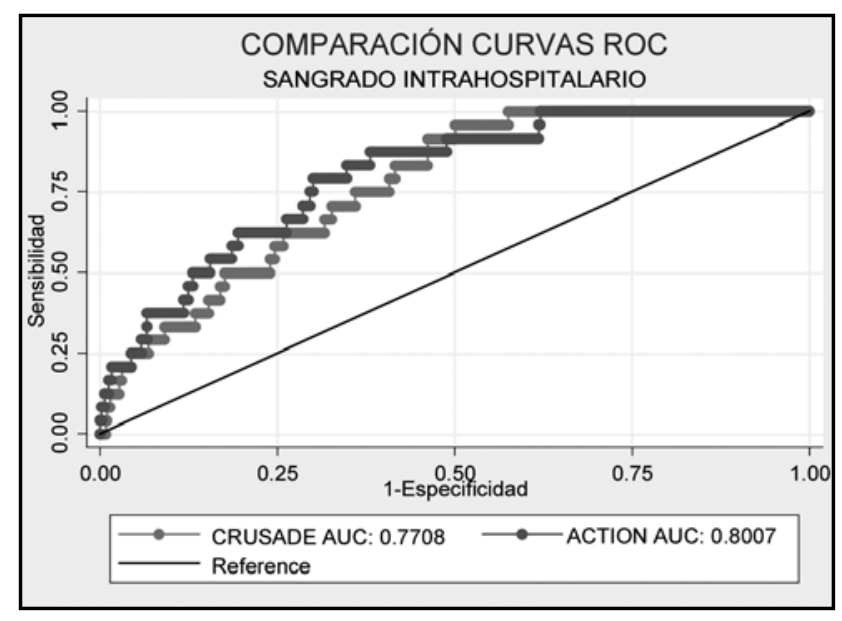

Figura 3. Comparación de curvas ROC para predicción de sangrado intrahospitalario de los puntajes CRUSADE y ACTION.

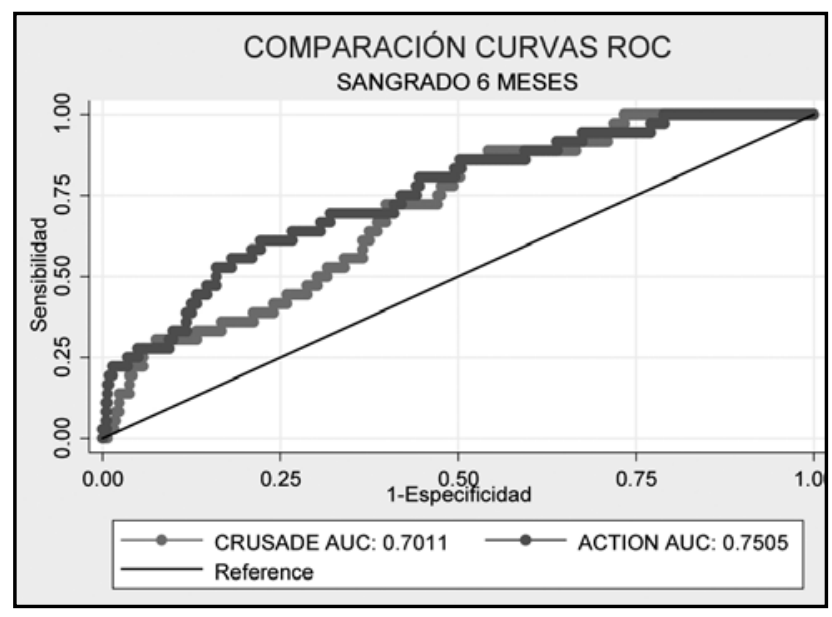

Figura 4. Comparación de curvas ROC para predicción de sangrado a los 30 días del alta hospitalaria de los puntajes CRUSADE y ACTION. 


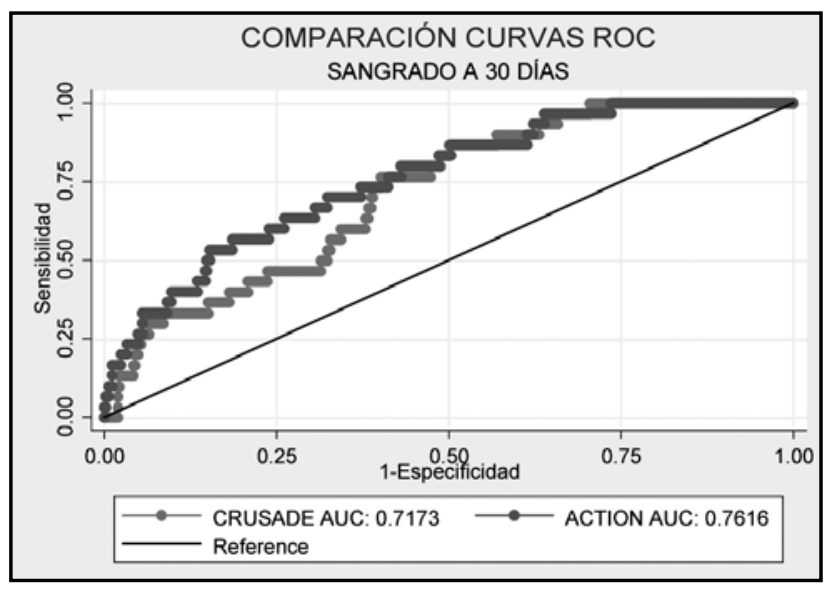

Figura 5. Comparación de curvas ROC para predicción de sangrado a los seis meses del alta hospitalaria de los puntajes CRUSADE y ACTION.

mente en el nivel sérico de creatinina encontrándose un valor promedio de $1.6 \mathrm{mg} / \mathrm{dL}$ comparado con $1.02 \mathrm{mg} / \mathrm{dL}$ y 1.1 $\mathrm{mg} / \mathrm{dL}$ de las escalas respectivas, pero con tasas de depuración de creatinina calculadas similares entre los pacientes incluidos en los diferentes estudios. En las demás variables demográficas podemos observar factores y perfiles de riesgo similares a las escalas que hemos validado.

La presencia de sangrado mayor fue $4.8 \%$, una tasa mucho menor a la reportada en cada uno de estos estudios en donde, por ejemplo, en la cohorte de derivación del CRUSADE la tasa de sangrado mayor fue de $9.4 \%$ y en la cohorte de validación la tasa de sangrado mayor fue $9.6 \%$, de la misma manera en el ACTION el grupo de pacientes con infarto de miocardio sin elevación del segmento ST la tasa de sangrado mayor fue de $10.2 \%$; probablemente este hallazgo de menor frecuencia de sangrado sea debido al uso de la técnica radial como vía de abordaje preferencial para el intervencionismo coronario, en lo cual se diferencia del abordaje reportadas en CRUSADE y ACTION, en donde la principal vía de acceso fue la arteria femoral.

Cuando comparamos las tasas de sangrado por cada uno de los grupos de riesgo según la estratificación por CRUSA$\mathrm{DE}$, excepto para el grupo de alto riesgo, el porcentaje de sangrado es mucho menor; en pacientes de muy bajo riesgo la tasa de sangrado fue de $2.3 \%$ comparado con $3.1 \%$ del CRUSADE, $4.6 \%$ vs $5.5 \%$ en bajo riesgo, $3.6 \%$ vs $8.6 \%$ en riesgo moderado, $12 \%$ vs $11.9 \%$ en alto riesgo y del $11.4 \%$ vs $19.5 \%$ en el grupo de muy alto riesgo, respectivamente. Para el ACTION el porcentaje de tasa de sangrado también fue mucho menor en nuestra población según la clasificación de cada uno de los grupos de riesgo.

Una de las principales consideraciones al momento de enfrentarnos al manejo de pacientes con un SCASEST será definir su riesgo basal individual de eventos isquémicos así como también de sus posibles complicaciones hemorrágicas. Es por esta razón que las guías de manejo del American College of Cardiology (ACC), la American Heart Association (AHA), European Cardiology Society (ECS), recomiendan la estratificación del riesgo de cada paciente para guiar la terapia (8). En el estudio ACUITY los pacientes con sangrado mayor tenían una mortalidad a 30 días significativamente mayor $(7.3 \%$ vs $1.2 \%, \mathrm{p}<0.0001)$. El sangrado mayor fue un predictor independiente de mortalidad a 30 días (OR 7.55, $95 \%$ IC 4.68 a 12.18, p<0.0001) (7). Hallazgos similares fueron observados en análisis derivados del estudio OASIS, en el cual la presencia de sangrado mayor incrementó de forma significativa el riesgo de mortalidad durante los primeros 30 días de seguimiento (6). En el registro GRACE se observó riesgo significativamente mayor de sangrado en pacientes de edad avanzada, sexo femenino, historia de sangrado previo y con insuficiencia renal; luego de ajustar por posibles variables confusoras la presencia de sangrado mayor fue asociada significativamente con el incremento del riesgo de mortalidad intrahospitalaria (OR 1.64, 95\% IC 1.18-2.28) (18). Recientemente Mendoza y colaboradores valoraron el rendimiento clínico de la escala CRUSADE en una población similar de pacientes, demostrando una apropiada predicción del riesgo de sangrado según el nivel de estratificación, con una frecuencia de sangrado significativamente mayor (18.18\%) que nuestra cohorte, enfocándola como prueba diagnóstica, para reportar sus características operativas (12); puede ser una metodología válida, aunque no se traduce directamente en utilidad clínica como se intuye, al igual que el área bajo la curva (19). En

Tabla 6. Calibración y capacidad discriminativa de los modelos de predicción CRUSADE y ACTION intrahospitalario, a 30 días y a los seis meses para sangrado.

\begin{tabular}{|c|c|c|c|c|c|c|}
\hline Intahospitalario & $\log M V^{*}$ & Seudo $\mathbf{R}^{2}$ & $\mathbf{H L}^{* *}, \mathbf{p}$ & AUC-ROC**** & IC $95 \%$ & $\mathbf{p}$ \\
\hline CRUSADE & -85.32 & 0.1170 & 0.6330 & 0.7708 & $0.69-0.84$ & 0.19 \\
\hline ACTION & -81.75 & 0.1540 & 0.7123 & 0.8007 & $0.72-0.87$ & \\
\hline \multicolumn{7}{|l|}{1 Mes } \\
\hline CRUSADE & -103.185 & 0.081 & 0.3534 & 0.71 & 0.63-0.79 & 0.0829 \\
\hline ACTION & -97.87 & 0.1283 & 0.5008 & 0.76 & $0.67-0.84$ & \\
\hline \multicolumn{7}{|l|}{ Seis meses } \\
\hline CRUSADE & -116.78 & 0.0752 & 0.1527 & 0.70 & $0.62-0.77$ & 0.0937 \\
\hline ACTION & -211.57 & 0.0639 & 0.8110 & 0.75 & $0.66-0.83$ & \\
\hline
\end{tabular}


una cohorte retrospectiva García y colaboradores describen pobre discriminación de la escala CRUSADE (AUC-ROC 0,61 IC 95\% 0.46-0,76) con adecuada calibración, también con altas tasas de sangrado (13), diferente a lo encontrado en nuestro estudio.

En el análisis bivariado se documentó aumento del riesgo de mortalidad entre los pacientes con sangrado significativo, especialmente durante el seguimiento a seis meses donde el OR fue de 5.316 con una p estadísticamente significativa para el presente análisis. Este hallazgo concuerda con los reportados en el CRUSADE en el cual también se apreció aumento de la mortalidad entre quienes presentaron y no presentaron sangrado significativo, en cada uno de los quintiles de riesgo, sin embargo, esta asociación se encontró intrahospitalaria. En el presente análisis el OR intrahospitalario es 1.007 con una p que no fue estadísticamente significativa y un intervalo de confianza amplio, probablemente por el número de pacientes y desenlaces incluidos.

Las dos escalas de riesgo de sangrado han mostrado una buena calibración y discriminación en nuestra serie, como se demostró de acuerdo al estadístico de Hosmer Lemeshow con un curva ROC AUC 0.77 para CRUSADE y 0.80 para ACTION en sangrado intrahospitalario, 0.70 y 0.75 a 30 días y 0.71 y 0.76 a seis meses de seguimiento, respectivamente. Los resultados reportados en el estudio CRUSADE fueron de 0.71 para la cohorte de derivación y de 0.70 para la cohorte de validación, a su vez en el estudio ACTION se reportó en 0.73 para derivación y 0.71 en validación; sin embargo esta última escala parece mejorar su precisión cuando se utiliza acceso radial (15). Igualmente, las dos escalas de riesgo de sangrado se corresponden con las tasas de sangrado observado, con incremento en la frecuencia conjuntamente con el aumento en la categoría de riesgo.

En un reciente análisis, se tomaron tres puntajes de riesgo para predicción de sangrado CRUSADE (9), ACTION(10) y ACUITY-HORIZONS(20); todos fueron llevados a intervención coronaria percutánea primaria predominantemente por vía transradial; los resultados mostraron una buena discriminación del riesgo de sangrado (AUC ROC 0.77, 0,78 y 0.70 respectivamente). Los puntajes CRUSADE y ACTION presentaron una mejor capacidad de predicción que la escala de riesgo ACUITY-HORINZONS ( $\mathrm{z}=3.89$, $\mathrm{p}$-valor $=0.0001$ y z $=0.63$, p-valor $=0.0004$, respectivamente) (21).

Las dos escalas de riesgo pueden ser una herramienta útil en nuestra población para identificar pacientes con un SCASEST llevados a una estrategia de intervención percutánea temprana con alto de riesgo de sangrado y ayudar al clínico a seleccionar la terapia antitrombótica más apropiada, la vía de acceso para el procedimiento diagnóstico y de intervención para cada caso en particular. Por lo tanto, con base en nuestros resultados creemos que cualquiera de las dos escalas puede ser empleada en el escenario de un SCASEST.

Uno de los aportes del presente análisis es el de la validación de dos escalas de riesgo para predecir sangrado en pacientes con SCASEST, predominantemente llevados a intervención transradial. La vía transradial para el intervencionismo coronario ha sido ampliamente utilizada en Europa y en nuestro medio, demostrando menores tasas de complicaciones hemorrágicas que el acceso femoral y permitiendo terapias antitrombóticas más agresivas $(22,23,24)$.

Limitaciones del estudio. La realización de estudios en un solo centro de alta complejidad podría no reflejar el comportamiento en la población general. Aunque el tamaño de muestra para validación de modelos de predicción sugiere que al menos tengan 100 desenlaces y 100 sin desenlaces, el requisito no aplica para la comparación de las escalas, obteniendo un buen poder para demostrar diferencias, en el caso de que las hubiere; a pesar de bajas tasas de sangrado en la actual cohorte se presentaron un numero suficiente de desenlaces para poder cumplir con el tamaño de muestra planeado. No esta claro el efecto que podría tener sobre la capacidad de discriminación de las escalas el uso de otros antiagregantes más potentes como prasugrel y ticagrelor o anticoagulantes como el fondaparinux o la bivaluridina que reportan menores tasas de sangrado $(25,26)$.

\section{Conclusiones}

Las escalas de riesgo CRUSADE y ACTION son herramientas útiles para la predicción del riesgo de sangrado en pacientes con SCASEST llevados a estratificación coronaria invasiva temprana, con adecuada capacidad de discriminación. Estos hallazgos sugieren el uso de la escala ACTION sobre la escala de riesgo CRUSADE, dado el AUC ROC obtenido en los diferentes escenarios analizados, aunque no se encontraron diferencias estadísticamente significativas.

\section{Referencias}

1. Steg PG, Huber K, Andreotti F, Arnesen H,Atar D, Badimon L, et al. Bleeding in acute coronary syndromes and percutaneous coronary interventions: position paper by the Working Group on Thrombosis of the European Society of Cardiology. Eur Heart J 2011; 32(15): 1854-1864.

2. Manoukian SV. Predictors and impact of bleeding complications in percutaneous coronary intervention, acute coronary syndromes, and ST-segment elevation myocardial infarction. Am J Cardiol 2009; 104(5 Suppl): 9C-15C.

3. Yusuf S, Zhao F, Mehta SR, Chrolavicius S, Tognoni G, Fox KK et al. Effects of clopidogrel in addition to Aspirin in patients with acute coronary syndromes without ST-segment elevation. $N$ Engl J Med. 2001; 345(7): 494-502.

4. Wiviott SD, Braunwald E, McCabe CH, Montalescot G, Ruzyllo W, Gottlieb $\mathbf{S}$, et al. Prasugrel versus clopidogrel in patients with acute coronary syndromes. N Engl J Med 2007; 357: 2001-15.

5. Wallentin L, Becker R, Budaj A, Cannon C, Emanuelsson H, Held C et al. Ticagrelor versus Clopidogrel in Patients with Acute Coronary Syndromes. $N$ Engl J Med 2009; 361: 1045-57.

6. Yusuf S, Mehta SR, Chrolavicius S, Afzal R, Pogue J, Granger CB, et al. Comparison of fondaparinux and enoxaparin in acute coronary syndromes. $N$ Engl J Med 2006; 354: 1464-76.

7. White HD, Chew DP, Hoekstra JW, Miller CD, Pollack C V., Feit F, et al Safety and Efficacy of Switching From Either Unfractionated Heparin or Enoxaparin to Bivalirudin in Patients With Non-ST-Segment Elevation Acute Coronary Syndromes Managed With an Invasive Strategy. Results From the ACUITY (Acute Catheterization and Urgen. J Am Coll Cardiol. 2008; 51: 1734-41.

8. Amsterdam E, Wenger N, Brindis R, Casey D, Ganiats T, et al. 2014 AHA ACC Guideline for the Management of Patients With Non-ST-Elevation Acute Coronary Syndromes. A Report of the American College of Cardiology/American Heart Association Task Force on Practice Guidelines. J Am Coll Cardiol 2014; 64: e139-e228.

9. Roffi M, Patrono C1, Collet JP, Mueller C, Valgimigli M, Andreotti F, et al 2015 ESC Guidelines for the management of acute coronary syndromes in patients 
presenting without persistent ST-segment elevation: Task Force for the Management of Acute Coronary Syndromes in Patients Presenting without Persistent ST-Segment Elevation of the European Society of Cardiology (ESC). Eur Heart J. 2016; 37(3): 267-315.

10. Subherwal S, Bach RG, Chen AY, Gage B, Rao S, Newby K, et al. Baseline risk of major bleeding in non-ST-segment-elevation myocardial infarction: the CRUSADE (Can Rapid risk stratification of Unstable angina patients Suppress ADverse outcomes with Early implementation of the ACC/AHA Guidelines) Bleeding Score. Circulation 2009; 119: 1873-1882.

11. Mathews R, Peterson ED, Chen AY, Wang T, Chin C, Fonarow G, et al. In-hospital major bleeding during ST-elevation and non-ST-elevation myocardial infarction care: derivation and validation of a model from the ACTION Registry(R)-GWTG. Am J Cardiol 2011; 107: 1136-1143.

12. Mendoza F, Jaramillo C, Ardila C. Evaluación del puntaje de sangrado "CRUSADE" como prueba diagnóstica para determinar sangrado mayor en pacientes con síndrome coronario agudo sin elevación del ST. Rev Colomb Cardiol 2014; 21: 13-23.

13. García A, Muñoz O, Urrea J, Burbano P. Validación de la escala CRUSADE para evaluar el riesgo de sangrado en pacientes con infarto agudo de miocardio sin elevación del segmento ST. Rev Colomb Cardiol 2015; 22(4): 169-178.

14.Abu-Assi E, Gracia-Acuna JM, Ferreira-Gonzalez I, et al. Evaluating the Performance of the Can Rapid Risk Stratification of Unstable Angina Patients Suppress Adverse Outcomes With Early Implementation of the ACC/AHA Guidelines (CRUSADE) bleeding score in a contemporary Spanish cohort of patients with non-ST-segment elevation acute myocardial infarction. Circulation 2010; 121: 2419-2426.

15. Taha S, D'Ascenzo F, Moretti C, Omedè P, Montefusco A, Bach RG, et al. Accuracy of bleeding scores for patients presenting with Myocardial Infarction: a meata-analysis of 9 studies abd 13759 patients. Postepy Kardiol Interwencyjnej 2015; 11(3): 182-190.

16. Thygesen K, Alpert J, Jaffe A, Simoons M, et al. Third universal definition of myocardial infarction. Eur Heart J 2012; 33: 2551-2567.

17. Cockcroft DW and Gault MH. Prediction of creatinine clearance from serum creatinine. Nephron 1976; 16: 31-41.
18. Moscucci M, Fox K a a, Cannon CP, Klein W, López-Sendón J, Montalescot $\mathbf{G}$, et al. Predictors of major bleeding in acute coronary syndromes: the Global Registry of Acute Coronary Events (GRACE). Eur Heart J. 2003; 24: 1815-23.

19. Vickers A, Van Calster B, Steyerberg E. Net benefit approaches to the evaluation of prediction models, molecular markers, and diagnostic tests. BMJ 2016; 352: i6.

20. Mehran R, Pocock SJ, Nikolsky E, et al. A risk score to predict bleeding in patients with acute coronary syndromes. J Am Coll Cardiol 2010; 55: 2556-2566.

21. Flores-Ríos X, Couto-Mallón D, Rodríguez-Garrido J, García-Guimaraes M, Gargallo-Fernández P, Piñón-Esteban P, et al. Comparison of the performance of the CRUSADE, ACUITY-HORIZONS, and ACTION bleeding risk scores in STEMI undergoing primary PCI: insights from a cohort of 1391 patients. Eur Heart Jou Acute Cardiovasc Care. 2013; 2: 19-26.

22.Jolly SS, Yusuf S, Cairns J, et al. Radial versus femoral access for coronary angiography and intervention in patients with acute coronary syndromes (RIVAL) a randomised, parallel group, multicentre trial. Lancet 2011; 377: 1409-1420.

23. Ferrante G, Rao SV, Jüni P, Da Costa BR, Reimers B5, Condorelli G, et al. Radial Versus Femoral Access for Coronary Interventions Across the Entire Spectrum of Patients With Coronary Artery Disease: A Meta-Analysis of Randomized Trials. JACC Cardiovasc Interv. 2016; S1936-8798(16): 30428-9.

24. Tamayo N, Vallejo F, Sénior J, Fernández A, Rodríguez A, Toro J. Eficacia y seguridad del acceso radial y femoral en pacientes con síndrome coronario agudo llevados a intervencionismo coronario. Redial -Registro de intervención radial-. Acta Med Colomb 2015; 40: 209-217.

25. Stone GW, White HD, Ohman EM, Bertrand ME, Lincoff AM, McLaurin BT, et al. Bivalirudin in patients with acute coronary syndromes undergoing percutaneous coronary intervention: a subgroup analysis from the Acute Catheterization and Urgent Intervention Triage strategy (ACUITY) trial. Lancet. 2007; 369(9565): 907-19.

26. Mehta SR, Granger CB, Eikelboom JW, Bassand JP, Wallentin L, Faxon DP, et al. Efficacy and safety of fondaparinux versus enoxaparin in patients with acute coronary syndromes undergoing percutaneous coronary intervention: results from the OASIS-5 trial. J Am Coll Cardiol. 2007; 50(18): 1742-51. 